\title{
Effects of Anaerobic Exercises on Cardiac Workload, Peripheral Resistance, and Lipid Index in Grade-I Hypertensive Young Adults
}

\author{
Ali Farhad \\ Ziauddin University, alifarhadtharwani@gmail.com \\ Sumaira Farooqui \\ Ziauddin University, sumairaimranfarooqui@gmail.com \\ Batool Hassan \\ Ziauddin University, batool.hassan@zu.edu.pk
}

Follow this and additional works at: https://nsuworks.nova.edu/ijahsp

Part of the Medicine and Health Sciences Commons

\section{Recommended Citation \\ Farhad A, Farooqui S, Hassan B. Effects of Anaerobic Exercises on Cardiac Workload, Peripheral Resistance, and Lipid Index in Grade-I Hypertensive Young Adults. The Internet Journal of Allied Health Sciences and Practice. 2021 Oct 01;19(4), Article 13.}

This Manuscript is brought to you for free and open access by the College of Health Care Sciences at NSUWorks. It has been accepted for inclusion in Internet Journal of Allied Health Sciences and Practice by an authorized editor of NSUWorks. For more information, please contact nsuworks@nova.edu. 


\title{
Effects of Anaerobic Exercises on Cardiac Workload, Peripheral Resistance, and Lipid Index in Grade-I Hypertensive Young Adults
}

\begin{abstract}
Purpose: Hypertension is the leading preventable risk factor for various cardiovascular diseases. With the soaring prevalence globally, there is a need to identify the non-pharmacological management for the maintenance of blood pressure. Hence this study is aimed to determine the impact of an-aerobic exercise in grade-I hypertensive young adults. Methods: A total of 92 participants were recruited from the OPD of a tertiary care hospital between the periods of 11 months (August 2017-June 2018) and were allocated in an anaerobic and aerobic group. The intervention was given based on ACSM, FITT protocol for 8 weeks. The pre- and post-systolic blood pressure, ankle brachial index ( $\mathrm{ABI}$ ), and lipid index were determined. Results: The results obtained from the study showed that eight weeks of prescribed exercises significantly improved systolic blood pressure, ankle brachial index, and lipid index of the hypertensive patients in both groups (Cl: $95 \%$, pConclusion: The study concluded that 8 weeks of supervised training programs were found to be effective in improving blood pressure, $\mathrm{ABI}$, and lipid index among grade-I hypertensive young adults. Both exercises regimes were found to be equally effective, however, in term of their impact on the dose-response relationship curve resisted exercises were found to be more potent than aerobic exercises as only 24 sessions of resisted exercises performed for 8 weeks provided an equally effective result as were obtained after 40 sessions of aerobic exercises.
\end{abstract}

\section{Author Bio(s)}

Dr Ali Farhad, MPhil is an Assistant Professor at Ziauddin College of Rehabilitation Sciences, Ziauddin University. Specialized in the field of Exercise Physiology

Dr Sumaira Farooqui, PhD is Associate Professor, Principal at Ziauddin College of Rehabilitation Sciences, Ziauddin University

Dr Batool Hassan, MPhil PhD scholar is Senior Lecturer at Ziauddin College of Rehabilitation Sciences, Ziauddin University. Specialized in the field of Exercise Physiology

\section{Acknowledgements}

First and Foremost, praises and thanks to Almighty Allah for His showers of blessings throughout my research work. I am sincerely grateful to the institution, Ziauddin College of Rehabilitation Sciences, for providing me the opportunity to undertake this research. I thank the management of the organization for their genuine support to complete the thesis. 


\title{
TISAHSP \\ The Internet Joumnal of Allied Health Sciences and Practice \\ Dedicated to allied health professional practice and education \\ Vol. 19 No. 4 ISSN 1540-580X
}

\section{Effects of Anaerobic Exercises on Cardiac Workload, Peripheral Resistance, and Lipid Index in Grade-I Hypertensive Young Adults}

\author{
Ali Farhad \\ Sumairia Farroqui \\ Batool Hassan \\ Ziauddin University \\ Pakistan
}

\begin{abstract}
Purpose: Hypertension is the leading preventable risk factor for various cardiovascular diseases. With the soaring prevalence globally, there is a need to identify the non-pharmacological management for the maintenance of blood pressure. Hence this study is aimed to determine the impact of an-aerobic exercise in grade-I hypertensive young adults. Methods: A total of 92 participants were recruited from the OPD of a tertiary care hospital between the periods of 11 months (August 2017-June 2018) and were allocated in an anaerobic and aerobic group. The intervention was given based on ACSM, FITT protocol for 8 weeks. The pre- and post-systolic blood pressure, ankle brachial index ( $A B I)$, and lipid index were determined. Results: The results obtained from the study showed that eight weeks of prescribed exercises significantly improved systolic blood pressure, ankle brachial index, and lipid index of the hypertensive patients in both groups $(\mathrm{Cl}: 95 \%, \mathrm{p}<0.05)$. Conclusion: The study concluded that 8 weeks of supervised training programs were found to be effective in improving blood pressure, $A B I$, and lipid index among grade-I hypertensive young adults. Both exercises regimes were found to be equally effective, however, in term of their impact on the dose-response relationship curve resisted exercises were found to be more potent than aerobic exercises as only 24 sessions of resisted exercises performed for 8 weeks provided an equally effective result as were obtained after 40 sessions of aerobic exercises.
\end{abstract}

Keywords: ankle brachial index, myocardial workload, hypertension, aerobic exercises, anaerobic exercises 
NCT: NCT04683523

(C) The Internet Journal of Allied Health Sciences and Practice, 2021 


\section{INTRODUCTION}

Substantial evidence has been provided by various epidemiological studies that hypertension (HTN) is the leading preventable risk factor for various cardiovascular diseases. ${ }^{1}$ According to American Heart Association (AHA), the prevalence of hypertension varies with age, and it is estimated that approximately $31.6 \%$ or nearly 23.78 million people around the globe are hypertensive, thus making hypertension a leading cause for cardiovascular morbidity. ${ }^{2}$ Apart from cardiovascular morbidity, HTN is also a leading cause of mortality, accounting for $13.5 \%$ of all deaths. ${ }^{3}$ According to a study conducted in 2015 , at present, the total direct cost attributed for the management of hypertension is US $\$ 130.7$ billion, which is estimated to increase up to US $\$ 389.9$ billion by 2030 ( 3 times from the present cost)..$^{4}$ Although the pharmacological advances have significantly improved HTN management, still a very few numbers of adult patients have their blood pressure under control. The goal that there would be a $50 \%$ reduction in HTN by 2010 has not yet been achieved. 5

Researchers continue to look for alternative methods for managing HTN. It has been concluded that among the various other risk factors for the development of hypertension, physical inactivity is the leading factor which can easily be modified by incorporating various lifestyle modifications. ${ }^{6}$ Hence, various national and international foundations and organization like the National Heart Foundation, the World Health Organization, the International Society of Hypertension, and American Society of Sports and Medicine advocated for an increase in physical activity and basic lifestyle modification as the first line of therapy for the treatment and the prevention of pre-hypertension, Grade I and Grade II hypertension ${ }^{7}$. Several research studies have suggested that for the management of grade II hypertension, pharmacological management is considered as the first-line therapy, and exercises are considered as an adjunct management strategy. The main purpose of this study is to determine the effects of exercise in the management of grade-1 hypertension.

Exercise prescription for the treatment of HTN is underestimated by the clinician and other health-related personnel mainly due to the ambiguity related to the dosage of the exercises regimes. ${ }^{8}$ However, with the advancement in exercise prescription determinants (i.e., frequency, intensity, time of exercise, and type of exercise [FITT protocol]), exercises are starting to be widely recommended. ${ }^{9}$ Physiologically, the impact of exercises on blood pressure is studied under two conditions 1) acute response and 2) the chronic response ${ }^{10}$.

Initially, exercises cause redistribution of cardiac output from the inactive part of the body towards active muscles taking part in exercises, thus causing an initial rise in systolic blood pressure (SBP) and cardiac output and a decrease in diastolic blood pressure (DBP) mainly due to a decrease in peripheral resistance. ${ }^{11}$ Whereas the chronic or the post-exercise response causes hypotension which may last up to 22 hours. This hypotensive response is mainly triggered by two essentials biomarkers -- nitric oxide and prostaglandins -- which are released from the stretched tunica intima of the arteries thereby inducing the extended vasodilation effects. ${ }^{12,13}$ The hypotensive response of exercises is mainly dependent on the dosage of exercises (primarily duration and type of exercise) thus giving rise to a different type of exercise protocol: aerobic, dynamic resisted and isometric combo (both aerobic and resisted). ${ }^{14,15}$ The post effects of every exercise regime on elevated blood pressure levels are different because of different physiological properties. The response of aerobic exercises as reported by Cornelissen et al in 2013 causes a reduction of 3.2 $\mathrm{mmHg}$ in SBP and $2.7 \mathrm{mmHg}$ in DBP after 6-52 weeks of an exercise regime. ${ }^{16}$ Similarly, Fargar et al found a maximum response of aerobic exercises in reduction of elevated blood pressure with 16 weeks of exercises causes a reduction in $6.9 \mathrm{mmHg}$ in SBP and $4.9 \mathrm{mmHg}$ in DBP. Studies on the response of resisted (anaerobic) exercises also provided results in favor of these exercises regimes where anaerobic exercises of 6-52 weeks and 8-52 weeks significantly reduced the elevated blood pressure by $2.7 \mathrm{mmHg}$ in SBP and $2.9 \mathrm{mmHg}$ in DBP and $1.8 \mathrm{mmHg}$ in SBP and $3.2 \mathrm{mmHg}$ in DBP respectively. ${ }^{17-18}$ The third and the most widely used protocol of exercises as suggested by the American College of Sports Medicine is a combined protocol of both aerobic and resisted exercises where 3-5 days of exercises per week for 16 consecutive weeks reduces blood pressure by $5 \mathrm{mmHg} .{ }^{19}$

The association between physical activity and blood pressure is dose dependent 20 An exercise duration of 60-90 minutes per week results in a more effective response in the alleviation of blood pressure than 31-60 minutes per week, and an exercise duration longer than 60-90 minutes per week does not effect blood pressure any further. ${ }^{21} \mathrm{It}$ is also reflected from multiple studies that in hypertensive patients, the long-term effects of exercise causes a reduction in systolic blood pressure mainly due to the vasodilation effects, whereas in healthy individuals, exercises cause an increase in end diastolic volume and reduction in the total peripheral resistance of the heart.

As evident from multiple studies, exercises have a beneficial impact on elevated blood pressure levels, and the response of exercise protocols varies due to the different physiological properties. The impact of each such exercises regime needs to be 
determined to determine the cost-effectiveness of exercises in term of the dosage and potency. Hence, the present study is aimed at determining the impact of anaerobic (resisted exercises) in grade-l hypertensive young adults.

\section{METHODS}

\section{Patients}

A total of 92 hypertensive patients with sedentary lifestyles were randomly recruited from the OPD of a tertiary care hospital between August 2017 and June 2018. Six patients withdrew from the study. The envelope method was used for randomization, 86 mutually exclusive envelopes were equally divided into two groups that were anaerobic and aerobic $(n=43)$, with the patients' ages between 20-40 years in each group. The approval was received from the Ethical Review Committee (ERC Ref \# 0180617AFMPT) of Ziauddin University in July 2017. A detailed description of the protocol along with pros and cons were discussed with the patients, and informed consent was signed by all participants. The protocol of exercise was based on the guidelines of the American College of Sports Medicine 201319 and the quantification of exercises was done using the FITT protocol. Group A was given intervention based on an anaerobic training protocol, and Group B was provided an aerobic exercise protocol.

\section{Procedures}

The frequency of exercises for patients recruited in group A was 3 days/week, the intensity of exercises was set to be $60 \%$ to $80 \%$ of 1 Repetition Maximum (RM), time was the duration to perform resistance exercises of 10 major muscles group of the body (biceps, triceps, trapezius, pectoralis major, latissimus dorsi, abdomen muscles, back extensors, hamstrings, quadriceps, and calf muscles), and resistance exercises were performed using dumbbells, TheraBand ${ }^{T M}$, and free weights. For patients recruited in group B, the frequency of exercises was set at 5 days/week, the intensity was set between $60 \%$ to $80 \%$ of maximum heart rate (calculated using Karvonen method), time was 30 minutes of aerobic training, and the type of exercises were treadmill or static cycling. Both the groups were given intervention for 8 weeks (Figure 1) under the supervision of a qualified physical therapist at the rehabilitation department of Ziauddin Hospital. All recruited patients were advised to adhere only to the given exercise protocol. The outcome measures were evaluated through blood pressure, ankle brachial index, and lipid profile.

\section{Inclusion/ Exclusion Criteria}

Screening of the participants was done based on the Physical Activity Readiness Questionnaire (PAR-Q and You form). Those who were found fit according to the questionnaire were further screened according to the inclusion and exclusion criteria.

Only patients with diagnosed grade-I hypertension were included in the study. All the participants were young adult's age between 20-40 years. The participants were initially scrutinized using Doppler ultrasound for the presence of peripheral artery disease and only those that had an ABI between 0.7-0.8 were included in the study. All those patients having any cardiovascular disorder or who declined to take part in the study were excluded from the trial.

\section{Exercise Termination Criteria ${ }^{22}$}

The exercise was prematurely terminated if any one of the following events occurred:

- A rise in heart rate above the upper limit of the targeted heart rate

- Oxygen saturation of less than $90 \%$

- Borg Rate of perceived exertion scale > 8, and

- At the request of the patient

\section{Outcome Measures \\ Blood Pressure}

Systolic and diastolic blood pressure of the participants was measured using a handcuff mercury sphygmomanometer. The blood pressure was measured before and after the completion of 8 weeks of the training protocol.

\section{Ankle Brachial Index}

The Ankle Brachial Index of the participants was measured using Huntleigh Dopplex MD2 Hand Doppler ultrasound. The readings were taken before and after the completion of 8 weeks of the training protocol.

\section{Lipid Index}

A blood serum lipid profile test was performed for HDL \& LDL values at the laboratory of Ziauddin University Hospital. The participants were asked to perform 12 hours of fasting before the test; the protocol includes the use of Roche/ BoehringerMannheim Diagnostic reagents. Apolipoprotein B-100 (apo B) was extracted from the blood serum that was analyzed to determine the blood values before and after completion of 8 weeks of the training protocol. 


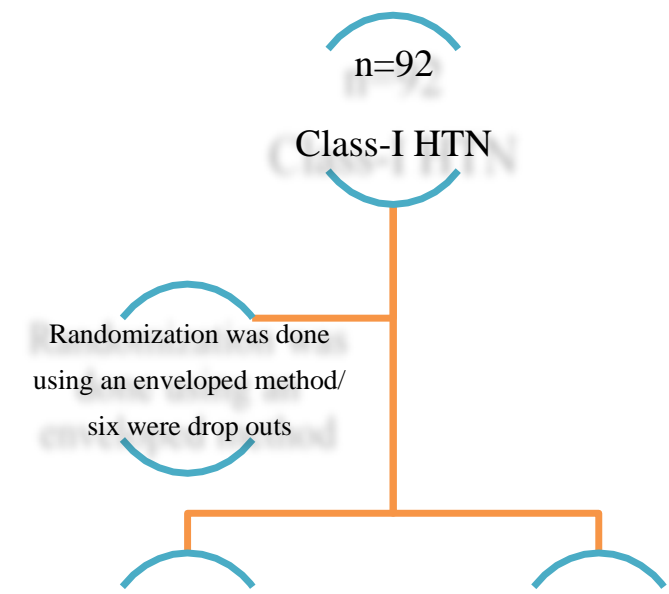

Group A n=43

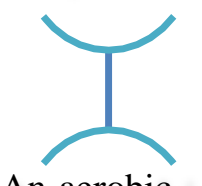

An-aerobic

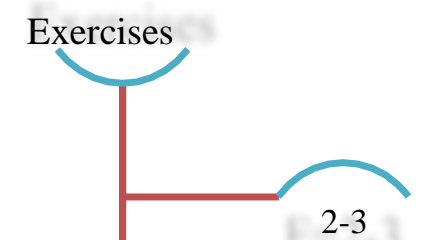

Days per

week

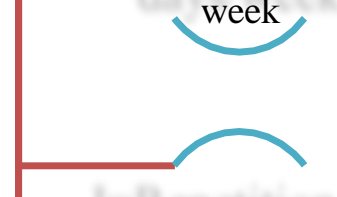

$60-80 \%$ of RM
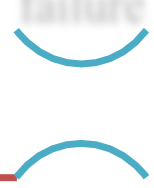

$\mathrm{T}=$ Time to complete set per major muscle

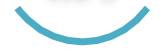

$\mathrm{T}=$ therabands and free weights

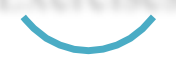

Group B n=43

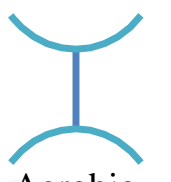

Aerobic

Exercises

5-6

Days per

week

$60-80 \%$ of

MHR

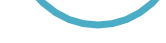

$\mathrm{T}=30 \mathrm{~min}$

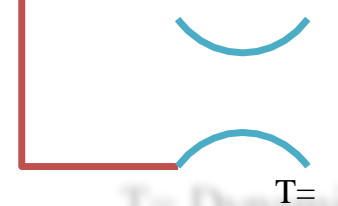

Treadmill/cycling

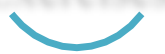

Figure 1. Exercise prescription based on ASCM guidelines (FITT Protocol) 


\section{Plan of Analysis}

1. Data was analyzed using medcalc statistical software

2. The pre- and post-test analysis was analyzed by using paired t-test as the data is not normally distributed

3. Level of significance was considered to be 0.05 with $95 \%$ of $\mathrm{Cl}$

\section{RESULTS}

The study included 92 grade I hypertensive young adult that were divided into two groups; 86 patients completed the study. Fiftysix patients were male and 30 were female with a mean age of $30 \pm 4.1$ in the anaerobic group and $34 \pm 3.2$ in the aerobic group. The baseline characteristics of patients are depicted in Table 1.

Table 1. Demographic details of the study participants

\begin{tabular}{|c|c|c|}
\hline Variables & Anaerobic & Aerobic \\
\hline $\mathbf{N}$ & 43 & 43 \\
\hline Age in years & $30 \pm 4.1$ & $34 \pm 3.2$ \\
\hline \multirow{2}{*}{ Gender } & 31 males & 25 male \\
\hline Height & 12 females & 18 females \\
\hline Weight & $5^{\prime} 6^{\prime}$ & $5^{\prime} 4^{\prime \prime}$ \\
\hline BMI & $74 \pm 42$ & $77 \pm 30$ \\
\hline
\end{tabular}

Within group analysis of the effects of exercises on the blood pressure was determined by using a paired t-test with a $95 \% \mathrm{Cl}$. The normality of the data was identified using a skewness and kurtosis test that confirmed the data was within the range of the normal distribution of the curve $( \pm 1.96)$. The results revealed that after eight weeks of exercise intervention, the average systolic blood pressure of the patients was reduced to $138 \pm 4.29$ from $149 \pm 5.22$ in the anaerobic group and $123 \pm 3.67$ from $150 \pm 4.85$ in the aerobic group. The detailed description of all variables is depicted in table 2.

Further between groups analysis was performed using an independent t-test at a $95 \% \mathrm{Cl}$ with the value of alpha at 0.05 . The results revealed that a significant difference in SBP was observed between both the groups; $p<0.05$ with aerobic exercises showing better improvement with a mean difference of $27 \pm 1.1$ as compared to anaerobic group with a mean difference of $11 \pm 1.2$.

The observed mean difference at $5 \%$ level of significance and a $95 \% \mathrm{Cl}$ was $0.05 \pm 0.001$ ( $95 \%$ of $\mathrm{Cl}, 0.02$ to 0.08 ) for Ankle Brachial Index showing significant improvement in favor of anaerobic group (mean;0.94) when compared to aerobic group (mean;0.89).

A significant mean difference of $p<0.05$ was found between the two groups that suggested the effectiveness of resistance exercises protocol over aerobic protocol in lowering the lipid index with a mean difference of $1.04 \pm 0.12$ and $0.65 \pm 0.01$ for anaerobic and aerobic group respectively. 
Table 2. Within group analysis using paired t test of SBP, $A B I$ and Lipid Index

\begin{tabular}{|c|c|c|c|c|c|}
\hline Groups & $\mathrm{n}$ & $\begin{array}{c}\text { Baseline } \\
\text { Mean } \pm S D\end{array}$ & $\begin{array}{c}\text { Eight weeks } \\
\text { Mean } \pm S D\end{array}$ & $M D \pm S D$ & $\begin{array}{l}\text { p value } \\
(<0.05)\end{array}$ \\
\hline \multicolumn{6}{|l|}{ SBP } \\
\hline Anaerobic & 46 & $149 \pm 5.22$ & $138 \pm 4.29$ & $11 \pm 1.2$ & \multirow{2}{*}{$<0.05$} \\
\hline Aerobic & 46 & $150 \pm 4.85$ & $123 \pm 3.67$ & $27 \pm 1.1$ & \\
\hline \multicolumn{6}{|c|}{ Ankle Brachial Index } \\
\hline Anaerobic & 46 & $0.72 \pm 0.04$ & $0.94 \pm 0.08$ & $0.22 \pm 0.01$ & \multirow{2}{*}{$<0.05$} \\
\hline Aerobic & 46 & $0.76 \pm 0.05$ & $0.89 \pm 0.06$ & $0.09 \pm 0.002$ & \\
\hline \multicolumn{6}{|l|}{ Lipid Index } \\
\hline Anaerobic & 46 & $2.93 \pm 0.26$ & $1.89 \pm 0.25$ & $1.04 \pm 0.12$ & \multirow{2}{*}{$<0.05$} \\
\hline Aerobic & 46 & $2.78 \pm 0.27$ & $2.13 \pm 0.3$ & $0.65 \pm 0.01$ & \\
\hline
\end{tabular}

\section{DISCUSSION}

The present study revealed that aerobic and anaerobic exercises were found to be effective in the management of grade-I hypertension with 8 weeks of exercise-based intervention of ACSM; the FITT protocol not only helped in reducing the systolic blood pressure of the participants but was also found to be effective in the management of $A B I$ and lipid profile of the patients. This is in contrast to the study by Salehi et al. in 2017 where it was reported that 8-week of resistance exercise did not lead to a significant decrease in the mean lipid profile. ${ }^{27}$

The results of the present study were in line with the result of Lee et al in which it was concluded that a longer duration exercise program reduces the systolic and diastolic blood pressure to around $3.2 \mathrm{mmHg}$ and $2.5 \mathrm{mmHg}$ respectively. ${ }^{9} \mathrm{Jacobson}$ et al found that 12 weeks of upper limb exercises improve the walking distance among the patients with leg pain. ${ }^{28}$ Similar findings were also observed by Mc Dermot et al in 2009 and 2014 where it was found that supervised exercise regimes improved the six-minute walk test among patients with and without claudication 29,30 Moreover, the impact of aerobic and anaerobic exercises on $\mathrm{MVO}_{2}$ was manifest from the fact that exercises induce the secretion of nitric oxide and prostaglandins, the two most important biomarkers of the body from the tunica intima of the arteries, causing a prolong post exercises vasodilation thereby reducing the workload of the heart during rest. ${ }^{11}$ Cornelissen and Smart in 2013, Kelley in 2010, and Owen et al in 2010 provided evidence in favor of resisted exercises in improving the systolic and diastolic blood pressure in which the maximum reduction in blood pressure as observed was $13.5 \mathrm{mmHg}$ in SBP and $7.8 \mathrm{mmHg}$ in DBP after $8-10$ weeks of exercise session with a frequency of 3 days per week. ${ }^{18,31,32}$

Fagard and Cornelissen in 2007 and Cornelissen et al in 2013 found that aerobic exercises also played a vital role in the management of blood pressure. ${ }^{16,17}$ Both reported a maximum reduction of $6.9 \mathrm{mmHg}$ and $4.9 \mathrm{mmHg}$ in systolic and diastolic blood pressure respectively after the completion of 16 weeks of exercise session performed with a frequency of 3 days/week. This suggests that although the effects of both the exercises protocol were the same and both were found to be equally effective in reducing the systolic and the diastolic blood pressure, but in term of efficacy and potency, the effects of resisted (anaerobic exercises) were greater than aerobic exercises. However, the question was, were resisted exercises safe? It was generally observed that resisted exercises were to be recommended only after assessing the general ability of the participants, and it was suggested that the criteria of measuring intensity for resisted training should be strictly followed as per international guidelines like ACSM, Joint National Committee, and American Heart Association, etc.

\section{Limitation}

No baseline characteristics of diet or physical activity were recorded, nor were other possible influences on the outcome measures noted. 


\section{CONCLUSION}

The study concluded that 8 weeks of supervised training programs were found to be effective in improving blood pressure, ABI, and lipid profile among grade-l hypertensive young adults. Both exercise regimes (aerobic and anaerobic) were found to be equally effective.

\section{Conflict of Interest Statement: No conflict of interest}

Funding Disclosure: There are no financial conflicts of interest to disclose

Acknowledgment: First and foremost, praises and thanks to Almighty Allah for His showers of blessings throughout my research work. I would like to acknowledge my deepest gratitude and render the warmest thanks to my research supervisor, Associate Prof. Dr. Sumaira Farooqui, who made this work possible. I am sincerely grateful to the institution, Ziauddin College of Rehabilitation Sciences, for providing me the opportunity to undertake this research. I thank the management of the organization for their genuine support to complete the thesis.

\section{REFERENCES}

1. Kumral ZN, Sener G, Ozgur S, Koc M, Suleymanoglu S, Hurdag C, Yegen BC. Regular exercise alleviates renovascular hypertension-induced cardiac/endothelial dysfunction and oxidative injury in rats. J Physiol Pharmacol. 2016 Feb 1;67(1):45-55.

2. Carey RM, Whelton PK. Prevention, detection, evaluation, and management of high blood pressure in adults: synopsis of the 2017 American College of Cardiology/American Heart Association hypertension guideline. Annals of Internal Medicine. 2018 Jan 23.

3. Brook RD, Appel LJ, Rubenfire M, Ogedegbe G, Bisognano JD, Elliott WJ, Fuchs FD, Hughes JW, Lackland DT, Staffileno BA, Townsend RR. Beyond medications and diet: alternative approaches to lowering blood pressure: a scientific statement from the American Heart Association. Hypertension. 2013 Jan 1:HYP-0b013e318293645f

4. Pescatello LS, MacDonald HV, Ash GI, Lamberti LM, Farquhar WB, Arena R, Johnson BT. Assessing the existing professional exercise recommendations for hypertension: a review and recommendations for future research priorities. InMayo Clinic Proceedings 2015 Jun 1 (Vol. 90, No. 6, pp. 801-812). Elsevier.

5. Yeh GY, Wang C, Wayne PM, Phillips RS. The effect of tai chi exercise on blood pressure: a systematic review. Preventive Cardiology. 2008 Mar 1;11(2):82-9.

6. Rossi A, Moullec G, Lavoie KL, Bacon SL. Resistance training, blood pressure, and meta-analyses. Hypertension. 2012 Mar 1;59(3):e22-3.

7. Owen A, Wiles J, Swaine I. Effect of isometric exercise on resting blood pressure: a meta analysis. J Hum Hypertens. 2010;24(12):796-800. doi:10.1038/jhh.2010.13

8. Pescatello LS, MacDonald HV, Lamberti L, Johnson BT. Exercise for hypertension: a prescription update integrating existing recommendations with emerging research. Curr Hypertens Rep. 2015 Nov 1;17(11):87.

9. Ghadieh AS, Saab B. Evidence for exercise training in the management of hypertension in adults. Can Fam Physician. 2015 Mar 1;61(3):233-9.

10. Semlitsch T, Jeitler K, Hemkens LG, Horvath K, Nagele E, Schuermann C, Pignitter N, Herrmann KH, Waffenschmidt $\mathrm{S}$, Siebenhofer A. Increasing physical activity for the treatment of hypertension: a systematic review and meta-analysis. Sports Medicine. 2013 Oct 1;43(10):1009-23.

11. Ruivo JA, Alcântara P. Hypertension and exercise. Rev Port Cardiol (English Edition). 2012 Feb 1;31(2):151-8.

12. Eicher JD, Maresh CM, Tsongalis GJ, Thompson PD, Pescatello LS. The additive blood pressure lowering effects of exercise intensity on post-exercise hypotension. American Heart Journal. 2010 Sep 1;160(3):513-20.

13. Chrysant SG. Current evidence on the hemodynamic and blood pressure effects of isometric exercise in normotensive and hypertensive persons. The Journal of Clinical Hypertension. 2010 Sep 1;12(9):721-6.

14. Pescatello LS. Exercise and hypertension: recent advances in exercise prescription. Current Hypertension Reports. 2005 Aug 1;7(4):281-6

15. Ghadieh AS, Saab B. Evidence for exercise training in the management of hypertension in adults. Can Fam Physician. 2015;61:233-9.

16. Cornelissen VA, Buys R, Smart NA. Endurance exercise beneficially affects ambulatory blood pressure: a systematic review and meta-analysis. Journal of Hypertension. 2013 Apr 1;31(4):639-48.

17. Fagard RH, Cornelissen VA. Effect of exercise on blood pressure control in hypertensive patients. European Journal of Cardiovascular Prevention \& Rehabilitation. 2007 Feb;14(1):12-7. 
18. Cornelissen VA, Smart NA. Exercise training for blood pressure: a systematic review and meta-analysis. Journal of the American Heart Association. 2013 Feb 22;2(1):e004473.

19. American College of Sports Medicine, editor. ACSM's health-related physical fitness assessment manual. Lippincott Williams \& Wilkins; 2013 Jan 21.

20. Weber MA, Schiffrin EL, White WB, Mann S, Lindholm LH, Kenerson JG, Flack JM, Carter BL, Materson BJ, Ram CV, Cohen DL. Clinical practice guidelines for the management of hypertension in the community. The Journal of Clinical Hypertension. 2014 Jan 1;16(1):14-26.

21. Li W, Fetherston C, Medigovich K. Taoist Tai Chi for health: The characteristics and patterns of practice of Western Australian practitioners. Health Behaviour \& Public Health. 2015;5(1):1-7.

22. Stoller O, de Bruin ED, Schindelholz M, Schuster-Amft C, de Bie RA, Hunt KJ. Efficacy of feedback-controlled roboticsassisted treadmill exercise to improve cardiovascular fitness early after stroke: a randomized controlled pilot trial. Journal of Neurologic Physical Therapy. 2015 Jul;39(3):156.

23. Chuang SY, Sung SH, Cheng HM, Hsu PF, Chou P, Chen CH. Ankle-brachial index and brachial-ankle pulse wave velocity jointed to predict mortality in a community study. Ranking: http://gateway. webofknowledge. com/gateway/Gateway. cgi? GWVersion= 2\&SrcAuth= NHRI\&SrcApp= NHRI_IR\&KeyISSN= 0195-668X\&DestApp= IC2JCR. 2015.

24. Treat-Jacobson D, Bronas UG, Leon AS. Efficacy of arm-ergometry versus treadmill exercise training to improve walking distance in patients with claudication. Vascular Medicine. 2009 Aug;14(3):203-13.

25. McDermott MM, Ades P, Guralnik JM, Dyer A, Ferrucci L, Liu K, Nelson M, Lloyd-Jones D, Van Horn L, Garside D, Kibbe M. Treadmill exercise and resistance training in patients with peripheral arterial disease with and without intermittent claudication: a randomized controlled trial. JAMA. 2009 Jan 14;301(2):165-74.

26. McDermott MM, Guralnik JM, Criqui MH, Ferrucci L, Zhao L, Liu K, Domanchuk K, Spring B, Tian L, Kibbe M, Liao Y. Home-based walking exercise in peripheral artery disease: 12-month follow-up of the GOALS randomized trial. Journal of the American Heart Association. 2014 Jun 19;3(3):e000711.

27. Salehi Z, Salehi K, Moeini M, Kargarfard M, Sadeghi M. The effect of resistance exercise on lipid profile of coronary artery disease patients: a randomized clinical trial. Iranian Journal of Nursing and Midwifery Research. 2017 Mar;22(2):112.

28. Di Raimondo D, Musiari G, Miceli G, Arnao V, Pinto A. Preventive and therapeutic role of Muscle contraction against chronic diseases. Current Pharmaceutical Design. 2016 Aug 1;22(30):4686-99.

29. Owen A, Wiles J, Swaine I. Effect of isometric exercise on resting blood pressure: a meta analysis. Journal of Human Hypertension. 2010 Dec;24(12):796. 